\title{
Letter on Bilateral Fan-Shaped Septal Extension Struts in East Asian Augmentation Rhinoplasty
}

\author{
Junshui Zheng ${ }^{1} \cdot$ Zuguang Hua $^{2} \cdot$ Peng Wei $^{2}$
}

Received: 8 June 2021 / Accepted: 10 June 2021 / Published online: 12 July 2021

(C) Springer Science+Business Media, LLC, part of Springer Nature and International Society of Aesthetic Plastic Surgery 2021

Level of Evidence $V$ This journal requires that authors assign a level of evidence to each article. For a full description of these Evidence-Based Medicine Ratings, please refer to Table of Contents or online Instructions to Authors www.springer.com/00266.

Sir,

We read with great interest the article entitled "Bilateral Fan-Shaped Septal Extension Struts in East Asian Augmentation Rhinoplasty" by Wang $\mathrm{H}$ et al. [1] in Aesthetic Plastic Surgery. In this article, the authors delineated a bilateral septal extension strut (SES) with the "sandwiched" structure which functions as both columellar strut and septal extension graft. The authors performed a retrospective study of 52 patients who received augmentation rhinoplasty surgery using SES and showed overall good aesthetic results.

Although the authors demonstrated efficacy with the SES, it is hard to believe that the good results were attributed to this implant due to the lack of control group in this study. The columellar strut, the septal extension graft, and the dorsal onlay graft all affect the overall surgical effect.

The outer part of the rib cartilage was made as the SES after being split from the middle into two mirroring grafts. We noted a paper on comma-shaped columellar strut grafts

Peng Wei

dr_weipeng@163.com

1 Ningbo University School of Medicine, No. 818, Fenghua Street, Jiangbei District, Ningbo 315000, Zhejiang, People's Republic of China

2 Ningbo No. 1 Hospital, No. 59, Liuting Street, Haishu District, Ningbo 315000, Zhejiang, People's Republic of China published by the same team [2]. What are the differences between the two grafts for shaping the nasal tip? What are their indications?

The authors carved the lateral part of the costal cartilage into the SES, because the physiological curvature of this part helps shape the nasolabial angle. We would like to know how to further modify or adjust the curvature of the SES to simulate the upwarped nasal tip. Besides, we would like to know the appropriate graft curvature and influence factors in its carving.

\section{Declarations}

Conflict of interest The authors declare that they have no conflicts of interest to disclose.

Human and Animal Rights This article does not contain any studies with human participants or animals performed by any of the authors.

Informed consent For this type of study informed consent is not required.

\section{References}

1. Wang H, Wu L, Xu Y et al (2021) Bilateral fan-shaped septal extension struts in East Asian augmentation rhinoplasty. Aesthetic Plast Surg 45(2):652-660

2. You J, Wu L, Xu Y et al (2021) Comma-shaped columellar strut for nasal tip plasty in East Asian rhinoplasty. Aesthetic Plast Surg 45(1):244-251

Publisher's Note Springer Nature remains neutral with regard to jurisdictional claims in published maps and institutional affiliations. 